\title{
Prevalence of BCR-ABL T315I Mutation in Malaysian Patients with Imatinib-Resistant Chronic Myeloid Leukemia
}

\author{
Yuslina Mat Yusoff*, Zahidah Abu Seman, Norodiyah Othman, Nor Rizan \\ Kamaluddin, Ezalia Esa, Nor Amalina Zulkiply, Julia Abdullah, Zubaidah \\ Zakaria
}

\begin{abstract}
Objective: Chronic Myeloid Leukemia (CML) is caused by a reciprocal translocation between chromosomes 9 and 22, $\mathrm{t}(9 ; 22)$ (q34;q11) which encodes for the BCR-ABL fusion protein. Discovery of Imatinib Mesylate (IM) as first line therapy has brought tremendous improvement in the management of CML. However, emergence of point mutations within the BCR-ABL gene particularly T315I mutation, affects a common BCR-ABL kinase contact residue which impairs drug binding thus contribute to treatment resistance. This study aims to investigate the BCR-ABL T315I mutation in Malaysian patients with CML. Methods: A total of 285 patients diagnosed with CML were included in this study. Mutation detection was performed using qualitative real-time PCR (qPCR). Results: Fifteen out of 285 samples (5.26\%) were positive for T315I mutations after amplification with real-time PCR assay. From the total number of positive samples, six patients were in accelerated phase (AP), four in chronic phase (CP) and five in blast crisis (BC). Conclusion: Mutation testing is recommended for choosing various tyrosine kinase inhibitors (TKIs) to optimize outcomes for both cases of treatment failure or suboptimal response to imatinib. Therefore, detection of T315I mutation in CML patients are clinically useful in the selection of appropriate treatment strategies to prevent disease progression.
\end{abstract}

Keywords: Chronic Myeloid Leukemia- BCR/ABL gene- T315I mutation- tyrosine kinase inhibitor

Asian Pac J Cancer Prev, 19 (12), 3317-3320

\section{Introduction}

Chronic Myeloid Leukemia (CML) is associated with the Philadelphia chromosome, which is caused by a reciprocal translocation between chromosomes 9 and 22 (Kurzrock et al., 1988; Melo, 1996). The t(9;22) (q34;q11) translocation results in constitutively active breakpoint cluster region-Abelson (BCR-ABL) tyrosine kinase and plays a central role in the pathogenesis of CML (Baccarani et al., 2012). The use of tyrosine-kinase inhibitors (TKIs) to inhibit BCR-ABL protein in CML is one the major milestones in modern oncology. Imatinib Mesylate (IM) was approved as first line therapy for newly diagnosed CML in chronic phase and prolonged survival of CML patient (Hochhaus et al., 2009). It is a selective inhibitor of the BCR-ABL tyrosine kinase that binds competitively to the adenosine triphosphate (ATP) binding site of BCR-ABL tyrosine kinase (Nagar et al., 2002). IM binds to the BCR-ABL tyrosine kinase ATP binding site and lead to inhibition of the tyrosine autophosphorylation and, in turn, phosphorylation of its substrates, thereby preventing the enzyme activity of the tyrosine kinase protein semi-competitively (Kantarjian et al., 2010).
The occurrence of resistance mutations of the BCR-ABL1 kinase domain are the most common cause of IM treatment failure in CML patients by shifting its equilibrium toward the open or active conformation (Nagar et al., 2002). Among them, the 'gatekeeper' mutation, T315I, affects catalytic domain of ABL kinase and cause complete resistance to treatment (Gorre et al., 2001). The T315I mutation results in a threonine (T) to an isoleucine (I) change at amino acid position 315. The second-generation TKIs, including dasatinib and nilotinib, have been shown to inhibit most IM-resistant BCR-ABL mutations, except for T315I (Guilhot et al., 2007; Hochhaus et al., 2007; Kantarjian et al., 2007; Hughes et al., 2009; Muller et al., 2009). The inclusion of other treatment modalities such as moving to allogeneic stem cell transplantation, or testing an investigational compound is required in affected patients (Pavlu et al., 2011).

The understanding of BCR-ABL mutational status is very useful in order to optimize therapeutic decision. Mutations Screening for T315I mutation in all CML patients resistant to TKIs treatment should be performed as early as possible to detect the lowest levels of the mutant 
clone (Baccarani et al., 2009; Jones et al., 2009). This study aimed to determine the frequency of BCR-ABL T315I mutation in Malaysian patients with CML.

\section{Materials and Methods}

\section{Subjects}

A total of $285 \mathrm{CML}$ patients with IM failure were included in the study. They were recruited from various hospitals in Peninsular Malaysia, Sabah, and Sarawak in 2013 to 2016. These patients were those in chronic, accelerated or blast phase, which has been treated with IM (Gleevec, Novartis) as first line treatment. According to European LeukemiaNet recommendations, failure means that the patient should be moved to other treatments because the response is such that the patient showed significant risk of progression and death (Baccarani et al., 2015). Bone marrow aspirate or peripheral blood samples were obtained from these patients and screened for BCR-ABL T315I mutation.

\section{Genomic DNA Extraction}

Genomic DNA was extracted from peripheral blood using QiAmp ${ }^{\circledR}$ DNA Blood Mini Kit (Qiagen $\mathrm{GmbH}$, Hilden, Germany) according to manufacturer's instructions. The quantity and ratio of absorbance at $260 \mathrm{~nm}$ and $280 \mathrm{~nm}(\mathrm{~A} 260 / 280)$ of purified gDNA was determined with a spectrophotometer (Nanodrop, USA).

\section{Mutation analysis}

Samples were subjected to mutation analysis using qualitative qPCR AmoyDx BCR-ABL T315I kit (Amoy Diagnostics Co., LTD, China) according to manufacturer's recommendation. AmoyDx BCR-ABL T315I kit was performed in tubes, on an Applied Biosystems 7500 Real-time PCR Machine (Life Technologies, USA). Each sample was tested for the presence of T315I mutation. $2 \mathrm{ng}$ of gDNA was used per reaction and the reaction volume was $35 \mu 1$.

The cycling conditions consisted of an initial denaturation step at $95^{\circ} \mathrm{C}$ for $5 \mathrm{~min}$, followed by 15 cycles of $95^{\circ} \mathrm{C}$ for $25 \mathrm{~s}, 60^{\circ} \mathrm{C}$ for $20 \mathrm{~s}, 72^{\circ} \mathrm{C}$ for $20 \mathrm{~s}$, and a total of 31 cycles of $93{ }^{\circ} \mathrm{C}$ for $25 \mathrm{~s}, 56{ }^{\circ} \mathrm{C}$ for $35 \mathrm{~s}, 72{ }^{\circ} \mathrm{C}$ for $20 \mathrm{~s}$. For quality control, samples were processed together with one positive and one no template control (NTC) each time. If the amplification curve was not classic S-curve or if $\mathrm{Ct}$ value was $\geq 29$, the result was determined as wild type (mutation negative). This kit provides a highly sensitive molecular technique to detect as low as $1 \% \mathrm{BCR}-\mathrm{ABL}$ T315I mutation DNA in background of 99\% normal DNA at $5 \mathrm{ng}$ sample DNA amount, while ensuring that false negatives are minimized.

\section{Results}

The demographic profiles of these $285 \mathrm{CML}$ patients are shown in Tables 1. Following the patients' characteristics, $63.2 \%$ of patients were male and $36.8 \%$ were female. The age of patient varies between 2 to 81 years old with mean value 42.2 years old. From a total number of patients, $58.9 \%$ were Malays, $22.5 \%$ Chinese, $10.2 \%$ Indian and $8.4 \%$ other races.

Fifteen out of 285 samples $(5.26 \%)$ were positive for T315I mutations after amplification with real-time PCR assay, in which 13 samples were male (Table 1). Malay patients constitute the highest $(\mathrm{n}=9)$ followed by Chinese, Indian and other races (Table 1). From the total number of positive samples, six patients were in accelerated phase (AP), four in chronic phase (CP) and five in blast crisis (BC).

\section{Discussion}

BCR-ABL kinase domain mutations had been reported to occur in $50 \%$ of patients who develop resistance to IM (Jabbour et al., 2006). Identification of mutational analysis at IM failure is important as it can facilitate selection of the most appropriate therapy to prevent or overcome resistance (Bixby and Talpaz, 2009). The common clinically relevant imatinib-resistant mutations are G250E, Y253F/H, E255K/V, T315I, M351T and F359V (Soverini et al., 2006). Second-generation ABL TKIs dasatinib (Shah et al., 2004), nilotinib (Weisberg et al., 2005), bosutinib (Golas et al., 2003) and bafetinib (Kimura et al., 2005) were developed for the treatment of imatinib-resistant patients. Unfortunately, these second-generation drugs are ineffective against the T315I mutation (Kaleem et al., 2015). Therefore, a third-line TKIs ponatinib was introduced to overcome IM failure and further improve the inhibitory effect (O'Hare et al., 2009). Ponatinib has

Table1. Patient Demographic Data and T315I Mutation Status

\begin{tabular}{lccc}
\hline & $\begin{array}{c}\text { T315I positive } \\
\mathrm{n}=15\end{array}$ & $\begin{array}{c}\text { T315I negative } \\
\mathrm{n}=270\end{array}$ & $\begin{array}{c}\text { Total patients } \\
\mathrm{n}=285\end{array}$ \\
\hline $\begin{array}{l}\text { Age [mean (range)] (years) } \\
\text { Gender }\end{array}$ & $37.3(21-62)$ & $42.45(2-81)$ & $42.13(2-81)$ \\
$\quad$ Male [n (\%)] & $13(4.6)$ & $167(58.6)$ & $180(63.2)$ \\
Female [n (\%)] & $2(0.7)$ & $103(36.1)$ & $105(36.8)$ \\
Ethnicity & & & $168(58.9)$ \\
Malay [n (\%)] & $9(3.2)$ & $159(55.8)$ & $64(22.5)$ \\
Chinese [n (\%)] & $3(1.1)$ & $61(21.5)$ & $29(10.2)$ \\
Indian [n (\%)] & $2(0.7)$ & $27(9.5)$ & $24(8.4)$ \\
Others [n (\%)] & $1(0.4)$ & $23(8.1)$ & \\
\hline
\end{tabular}


high activity against ABL kinase point mutation including T315I by binding to the inactive conformation of the kinase domain in the murine ABL T315I (O'Hare et al., 2009; Cortes et al., 2012).

This study aimed to determine the frequency of T315I mutation in CML patients treated with IM. There are various techniques used to detect BCR-ABL mutations which will greatly influence the detection frequency and analytical sensitivity. It ranges from Sanger sequencing to denaturing high performance liquid chromatography, liquid bead array, pyrosequencing and mutation specific qPCR (Jones et al., 2009). In this study, we used qPCR technique using AmoyDx BCR-ABL T315I kit. This method is highly selective and sensitive in the detection of T315I mutation, in which it can identify mutant DNA in samples containing as little as $1 \%$ mutant DNA in a mixture with $99 \%$ wild-type DNA, while ensuring that false negatives are minimized.

The BCR-ABL kinase domain mutations that located in the IM binding domain (amino acids 315-317) are reported to have association with IM-resistance (Jones et al., 2009). These amino acids form a critical hydrogen bonds with IM. The substitution of threonine to isoleucine at residue 315 (T315I), thus impaired IM placement within the ATP-binding pocket of the BCR-ABL gene (Gorre et al., 2001; Nagar et al., 2002). The T315I is reported to be the most frequent mutation related to resistance to most second generation TKIs. Elnahass et al., (2013) reported that $3.57 \%(1 / 28)$ T315I mutation was detected in IM resistant patients. The findings from Dhahi et al., (2013) showed the frequency of T315I mutation was detected in 1 of $34,3.03 \%$ CML patients in accelerated phase after IM failure. Both studies were performed using allele-specific oliogonucleotide-PCR for mutation screening (Elnahass et al., 2013). A study conducted by Elias et al., (2012) reported that T315I mutation was detected in 8 of 40 cases $(20 \%)$ in Philadelphia chromosome $(\mathrm{Ph})$ positive CML patients who showed resistance to IM. In addition, Roche-Lestienne et al., (2002) revealed that $12.5 \%(3 / 24)$ of IM-resistant patients showed positive T315I. The T315I mutation was detected in $5.2 \%(10 / 191)$ CML patients in the current study. Recently, the study on IM-resistant CML patients showed two of twenty-two patients had T315I mutation (Yap et al., 2017). Our finding is in concomitant with a study conducted by other researchers in similar population (Elias et al., 2014). In their study, 7.2\% (9/125) T315I mutations were detected in Malaysian CML patients whom undergoing IM therapy.

The result of our study showed that $15 \mathrm{CML}$ patients with T315I point mutations were in $\operatorname{AP}(n=6)$ and acute $\mathrm{BC}(\mathrm{n}=5)$. T315I mutations were also detected in four CP CML patients, suggesting that point mutations in the ABL gene is not restricted to accelerated phase or acute blastic crisis patients (Roche-Lestienne et al., 2002). Therefore, mutation screening in CP CML is recommended in patients with inadequate initial response or those with evidence of loss of response. It is also recommended at the time of progression to accelerated or blast phase (Jones et al., 2009). In our setting, a T315I mutation should lead to consideration of allogeneic haematopoetic stem cell transplant.

In conclusion, BCR-ABL T315I mutation is the most severe form of tyrosine kinase domain (TKD) mutation as it causes decrease affinity for TKIs and thereby promotes shortening of patients' survival. Therefore, detection of T315I mutation by molecular techniques are clinically useful in the selection of appropriate treatment strategies to prevent disease progression in CML patients. Further study on the full spectrum of TKD mutations will be performed to look at different level of resistance in order to guide therapy in CML patients.

\section{Acknowledgements}

This work was supported by an operational budget from Haematology Unit, Cancer Research Centre, Institute for Medical Research. We would like to thank the Director General of Health Malaysia for his permission to publish this article. The authors would like to declare that there was no conflict of interest in this study.

\section{References}

Baccarani M, Castagnetti F, Gugliotta G, et al (2009). Response definitions and European Leukemianet Management recommendations. Best Pract Res Clin Haematol, 22, 331-41.

Baccarani M, Castagnetti F, Gugliotta G, et al (2015). A review of the European LeukemiaNet recommendations for the management of CML. Ann Hematol, 94, 141-7.

Baccarani M, Pileri S, Steegmann JL, et al (2012). Chronic myeloid leukemia: ESMO Clinical Practice Guidelines for diagnosis, treatment and follow-up. Ann Oncol, 23, vii72-vii7.

Bixby D, Talpaz M (2009). Mechanisms of resistance to tyrosine kinase inhibitors in chronic myeloid leukemia and recent therapeutic strategies to overcome resistance. Hematology Am Soc Hematol Educ Program, 10, 461-76.

Cortes JE, Kantarjian H, Shah NP, et al (2012). Ponatinib in refractory Philadelphia chromosome-positive leukemias. $N$ Engl J Med, 367, 2075-88.

Dhahi MAR, Matti BF, Fadel S (2013). Molecular screening for T315I and F317L resistance mutations in Iraqi chronic myeloid leukemia non-responders patients to imatinib. Cancer Clin Oncol, 2, 55-61.

Elias MH, Baba AA, Azlan H, et al (2014). BCR-ABL kinase domain mutations, including 2 novel mutations in imatinib resistant Malaysian chronic myeloid leukemia patients-Frequency and clinical outcome. Leuk Res, 38, 454-9.

Elias MH, Baba AA, Husin A, et al (2012). Contribution of BCR-ABL kinase domain mutations to imatinib mesylate resistance in Philadelphia chromosome positive Malaysian chronic myeloid leukemia patients. Hematol Rep, 4, e23.

Elnahass YH, Mahmoud HK, Ali FT, et al (2013). Abl Kinase Domain Mutations in Imatinib-treated Egyptian Patients with Chronic Myeloid Leukemia. J Leuk, 1, 1-8.

Golas JM, Arndt K, Etienne C, et al (2003). SKI-606, a 4-anilino-3-quinolinecarbonitrile dual inhibitor of Src and $\mathrm{Abl}$ kinases, is a potent antiproliferative agent against chronic myelogenous leukemia cells in culture and causes regression of K562 xenografts in nude mice. Cancer Res, 63, 375-81.

Gorre ME, Mohammed M, Ellwood K, et al (2001). Clinical resistance to STI- 571 cancer therapy caused by BCR-ABL

Asian Pacific Journal of Cancer Prevention, Vol 193319 
gene mutation or amplification. Science, 293, 876-80.

Guilhot F, Apperley J, Kim DW, et al (2007). Dasatinib induces significant hematologic and cytogenetic responses in patients with imatinib-resistant or -intolerant chronic myeloid leukemia in accelerated phase. Blood, 109, 4143-50.

Hochhaus A, Kantarjian HM, Baccarani M, et al (2007). Dasatinib induces notable hematologic and cytogenetic responses in chronic-phase chronic myeloid leukemia after failure of imatinib therapy. Blood, 109, 2303-9.

Hochhaus A, O'Brien SG, Guilhot F, et al (2009). Six-year follow-up of patients receiving imatinib for the first-line treatment of chronic myeloid leukemia. Leukemia, 23, 1054-61.

Hughes T, Saglio G, Branford S, et al (2009). Impact of baseline BCR-ABL mutations on response to nilotinib in patients with chronic myeloid leukemia in chronic phase. $J$ Clin Oncol, 27, 4204-10.

Jabbour E, Kantarjian H, Jones D, et al (2006). Frequency and clinical significance of BCR-ABL mutations in patients with chronic myeloid leukemia treated with imatinib mesylate. Leukemia, 20, 1767-73.

Jones D, Kamel-Reid S, Bahler D, et al (2009). Laboratory practice guidelines for detecting and reporting BCR-ABL drug resistance mutations in chronic myelogenous leukemia and acute lymphoblastic leukemia: a report of the Association for Molecular Pathology. J Mol Diagn, 11, 4-11.

Kaleem B, Shahab S, Ahmed N, et al (2015). Chronic myeloid leukemia - prognostic value of mutations. Asian Pac J Cancer Prev, 16, 7415-23.

Kantarjian HM, Cortes J, La Rosee P, et al (2010). Optimizing therapy for patients with chronic myelogenous leukemia in chronic phase. Cancer, 116, 1419-30.

Kantarjian HM, Giles F, Gattermann N, et al (2007). Nilotinib (formerly AMN107), a highly selective BCR-ABL tyrosine kinase inhibitor, is effective in patients with Philadelphia chromosome-positive chronic myelogenous leukemia in chronic phase following imatinib resistance and intolerance. Blood, 110, 3540-6.

Kimura S, Naito H, Segawa H, et al (2005). NS-187, a potent and selective dual Bcr-Abl/Lyn tyrosine kinase inhibitor, is a novel agent for imatinib-resistant leukemia. Blood, 106, 3948 .

Kurzrock R, Gutterman JU, Talpaz M (1988). The molecular genetics of Philadelphia chromosome-positive leukemias. N Engl J Med, 319, 990-8.

Machado MP, Tomaz JP, Lorand-Metze I, et al (2011). Monitoring of BCR-ABL levels in chronic myeloid leukemia patients treated with imatinib in the chronic phase - the importance of a major molecular response. Rev Bras Hematol Hemoter, 33, 211-5.

Melo JV (1996). The diversity of BCR-ABL fusion proteins and their relationship to leukemia phenotype. Blood, $\mathbf{8 8}$, 2375-84.

Muller MC, Cortes JE, Kim DW, et al (2009). Dasatinib treatment of chronic-phase chronic myeloid leukemia: analysis of responses according to preexisting BCR-ABL mutations. Blood, 114, 4944-53.

Nagar B, Bornmann WG, Pellicena P, et al (2002). Crystal structures of the kinase domain of c-Abl in complex with the small molecule inhibitors PD173955 and imatinib (STI-571). Cancer Res, 62, 4236-43.

O'Hare T, Shakespeare WC, Zhu X, et al (2009). AP24534, a Pan-BCR-ABL inhibitor for chronic myeloid leukemia, potently inhibits the T315I mutant and overcomes mutation-based resistance. Cancer Cell, 16, 401-12.

Pavlu J, Szydlo RM, Goldman JM, et al (2011). Three decades of transplantation for chronic myeloid leukemia: what have we learned?. Blood, 117, 755-63.

Roche-Lestienne C, Soenen-Cornu V, Grardel-Duflos N, et al (2002). Several types of mutations of the Abl gene can be found in chronic myeloid leukemia patients resistant to STI571, and they can pre-exist to the onset of treatment. Blood, 100, 1014-8.

Shah NP, Tran C, Lee FY, et al (2004). Overriding imatinib resistance with a novel ABL kinase inhibitor. Science, $\mathbf{3 0 5}$, 399-401.

Soverini S, Colarossi S, Gnani A, et al (2006). Contribution of ABL kinase domain mutations to imatinib resistance in different subsets of Philadelphia-positive patients: by the GIMEMA Working Party on Chronic Myeloid Leukemia. Clin Cancer Res, 12, 7374-9.

Weisberg E, Manley PW, Breitenstein W, et al (2005). Characterization of AMN107, a selective inhibitor of native and mutant Bcr-Abl. Cancer Cell, 7, 129-41.

Yap E, Tumian NR, Azma RZ, et al (2017). Primary imatinib resistance in chronic myeloid leukemia patients in a developing country: BCR-ABL kinase domain mutations or BCR-ABL independent mechanisms?. Malays J Pathol, 39, 107-13.

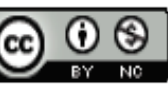

This work is licensed under a Creative Commons AttributionNon Commercial 4.0 International License. 